researchers, physicians and all healthcare workers who may become primarily exposed to such xenobiotics during their job tasks. The aim of this work is to discuss promising solutions provided by nanotechnology in medical fields, with a specific focus on critical aspects and research needs for occupational risk assessment and management in this emerging field.

Methods Pubmed, Scopus and ISI Web of Science databases were searched to identify studies addressing potential applications of nanoscale science, and technology for medical aims with attention focused on emerging occupational risks.

Results Favourable optical and chemical nanomaterial properties may enhance medical imaging, as well as molecular and gene diagnostics. Nano-carriers may improve bioavailability, and tissue specificity of drugs. Nanomaterials proved anti-bacterial and anti-viral properties, increased sensitivity to radiation therapy, and supported tissue repair. However, hazard identification of nano-medical formulations, exposure assessment, risk characterisation in lab and clinical settings, and possible risk management strategies for exposed workers resulted still almost unexplored.

Discussion Despite the many proposed advantages for nanomedical innovations, occupational risk assessment and management processes may take advantage from further research aimed to define the toxicological profile of differently characterised nano-formulations, to assess qualitative and quantitative exposure aspects related to the different phases of application, in ordinary work conditions and in case of accidental contacts, to identify biological exposure and early effect indicators to be potentially employed in well-organised health surveillance programs. Overall, this review highlights the importance to define adequate precautionary risk management strategies for workers, and occupational safety practices and policies, in order to develop a responsible consensus on nanotechnology in medicine.

\section{C RISK FACTORS OF VIOLENCE AGAINST HEALTHCARE AND SOCIAL WORKERS IN GERMANY}

A Nienhaus, S Steinke, A Kozak, A Schablon. University Clinics Hamburg Eppendorf. Centre for Health Service Research in Nursing, Germany

\subsection{6/oemed-2018-ICOHabstracts.933}

Introduction Healthcare and social welfare workers are confronted with violence of patients and clients. In a cross-sectional study the frequency and consequences of aggressive assaults on employees in the German healthcare and welfare system were investigated.

Methods At the workplace employees were asked to fill in a standardised questionnaire concerning the frequency and consequences of verbal and physical aggressions within the last year. The questionnaire was adopted from the Staff Observation Aggression Scale-Revised (SOAS-R).

Results 1943 employees from 81 different facilities participated in the survey (response rate 40\%). Verbal aggression was experienced by $75 \%$ and physical aggression by $55 \%$ of the participants. Both forms of aggression occurred more often in workshops and homes for persons with handicaps $(95 \%$ and $63 \%)$ than in nursing care $(59 \%$ and $56 \%)$ or hospitals.

(41\% and $43 \%) .39 \%$ of the employees were hit within the last 12 months. About one third of the participants (34\%) felt highly strained by recurring aggressions. 38\% reported that they were trained at the workplace for dealing with these critical incidents and $81 \%$ felt supported by their co-worker after such an incidence. De-escalation training and supervision had a positive effect on experienced stress (OR 0.6, 95\% CI: 0.4 to 0.8$)$.

Conclusion Violence towards nursing and healthcare personnel occurs frequently. Every third respondent felt severely stressed by violence and aggression. Occupational support provisions to prevent and provide aftercare for cases of violence and agression reduced the risk of incidences and of perceived stress. Research is needed on occupational support provisions that reduce the risk of staff experiencing verbal and physical violence and the stress that is associated with it.

\section{HANDLING OF HAZARDOUS DRUGS IN HEALTH CARE SETTINGS}

Rosa M Orriols*. CEO Occupational Health, Hospital Universitari Bellvitge-Institut Català de la Salut, Barcelona

\subsection{6/oemed-2018-ICOHabstracts. 934}

${ }^{1}$ Rogers B, ${ }^{2}$ McDiarmid M, ${ }^{3}$ Levy M, ${ }^{4}$ Orriols R

${ }^{1}$ University of North Carolina, Chapel Hill North Carolina, USA

${ }^{2}$ University of Maryland School of Medicine, Baltimore Maryland, USA

${ }^{3}$ Institute of Occupational and Environmental Medicine Rambam Medical Campus, Haifa, Israel

${ }^{4} \mathrm{ICOH}-\mathrm{SCOHHCW}$

\section{6a HEALTH EFFECTS OF HAZARDOUS DRUGS}

B Rogers. University of North Carolina, Chapel Hill, NC, USA

10.1136/oemed-2018-ICOHabstracts.935

Healthcare workers are exposed to numerous hazardous drugs including antineoplastic agents, antiviral drugs, hormones, and bioengineered/miscellaneous drugs. The National Institute for Occupational Safety and Health (NIOSH) in the U.S. has defined hazardous drugs as those that exhibit one or more of the following characteristics in animals or humans: carcinogenicity, teratogenicity, reproductive toxicity, organ toxicity at low does, genotoxicity, and toxic profiles of new drugs that mimic existing drugs. The actual risk to healthcare workers depends on drug toxicity, route of drug entry (e.g., inhalation, percutaneous, ingestion), and work practice handling and exposure and controls. Many of these drugs affect human cell systems through DNA damage, interference with cell growth, or may cause mutations. In considering the hazardous nature of the drug, NIOSH examines the dose for animal testing that results in reproductive or developmental toxic effects, any available human data with toxic effects, and those drugs requiring safe-handling practices as determined from the manufacturer. There are many drugs that are considered hazardous and all of these cannot be discussed here. However, examples will be provided as to effects of antineoplastic agents and non-antineoplastic agents. The issue of safety culture is always important in terms of prevention and control strategies and recognition of the harmful effects of these substances. 